\title{
Politiknetzwerke in der europäischen Integration: Zeitgeschichte und Politikwissenschaft im Dialog
}

\author{
Wolfram Kaiser*
}

Historiker, die sich mit der europäischen Zeitgeschichte nach 1945 befassen, sollten vor allem für die Zeit ab den 1960er Jahren die breite sozialwissenschaftliche Forschung nicht ignorieren. Mit der Ausnahme weniger Forscher wie Alan S. Milward ${ }^{1}$ und Wilfried Loth ${ }^{2}$ haben jedoch zumindest die meisten Historiker der europäischen Integration der ersten und zweiten Generation gerade dies konsequent getan. Sie haben Integration überwiegend sehr positivistisch und deskriptiv als diplomatischen Aushandlungsprozess beschrieben. Selbst Milward hat lange Zeit unreflektiert von ,nationalen Interessen“ gesprochen, bevor er diesen Terminus durch „,nationale Strategien“ ersetzte. ${ }^{3}$ Andererseits erschwert die Professionalisierung der politikwissenschaftlichen Forschung, die sich nach amerikanischem Vorbild immer mehr als ,political science" denn als ,political studies" geriert, zunehmend das wechselseitige Verständnis und die Anschlussfähigkeit zwischen Geschichts- und Politikwissenschaft. So erfolgt in der Politikwissenschaft mit der Ausnahme oftmals schematisch angewandter und empirisch dürftig belegter Konzepte wie dem des historischen Institutionalismus kaum mehr eine historische Verortung gegenwartsbezogener Forschung.

Dieser Beitrag soll eine Brücke über den so entstandenen, immer tieferen Graben zwischen zeitgeschichtlicher und politikwissenschaftlicher Forschung zur heutigen Europäischen Union bauen helfen. ${ }^{4}$ Historiker können nur mit sozialwissenschaftlich belesener und konzeptionell und methodisch präziser Forschung einen wesentlichen Beitrag zu dem hier vorgeschlagenen vertieften interdisziplinären Dialog leisten. ${ }^{5}$ Eine konzeptionell anspruchsvolle zeithistorische Forschung könnte sozialwissenschaftliche Konzepte und Annahmen über Politikprozesse in der Europäischen Union kritisch hinterfragen und - perspektivisch vor allem zu einem besseren Verständnis der temporalen Dimension der europäischen Integration beitragen: das heißt im Kontext dieses Beitrags vor allem dazu, inwieweit sich Regieren in der Europäischen Union von den Anfängen des Klein- oder Kerneuropas der 1951/52

Prof. Dr. Wolfram Kaiser, Universität Portsmouth; Europakolleg, Brügge.

1 Alan S. Milward/Frances M. B. Lynch/Federico Romero/Ruggero Ranieri/Vibeke Sørensen: The Frontier of National Sovereignty. History and theory 1945-1992, London 1993.

2 Wilfried Loth/Wolfgang Wessels (Hrsg.): Theorien europäischer Integration, Opladen 2001.

3 Alan S. Milward: The Rise and Fall of a National Strategy 1945-1963, London 2002, S. 6.

4 Vgl. hierzu auch aus beiden disziplinären Perspektiven mit Bezug auf die Analyse der Europäischen Union insgesamt Wolfram Kaiser: History meets Politics: Overcoming Interdisciplinary Volapük in Research on the EU, in: Journal of European Public Policy 2/2008, S. 300-313; Alex Warleigh-Lack: Interdisciplinarity in Research on the EU: Politics, history and prospects for collaboration, in: Wolfram Kaiser/Brigitte Leucht/Morten Rasmussen (Hrsg.): The History of the European Union. Origins of a trans- and supranational polity 1950-72, Abingdon/ New York 2009, S. 206-220; Alex Warleigh: In Defence of Intra-disciplinarity: ,European Studies', the ,New Regionalism‘ and the Issue of Democratisation, in: Cambridge Review of International Affairs 2/2004, S. 301318.

5 Vgl. Wolfram Kaiser/Brigitte Leucht/Morten Rasmussen: Origins of a European polity: A new research agenda for European Union history, in: Wolfram Kaiser/Brigitte Leucht/Morten Rasmussen (Hrsg.): The History of the European Union. Origins of a trans- and supranational polity 1950-72, Abingdon/New York 2009, S. 1-11; Wolfram Kaiser/Michael Gehler/Brigitte Leucht: Networks in Informal European Governance. Diachronic Perspectives on the European Union as a Multi-Level Polity, in: Michael Gehler/Wolfram Kaiser/Brigitte Leucht (Hrsg.): Netzwerke im europäischen Mehrebenensystem. Von 1945 bis zur Gegenwart, Wien/Köln/ Weimar 2009, S. 9-26. 
gegründeten Europäischen Gemeinschaft für Kohle und Stahl (EGKS) bis zum bisher noch nicht abschließend ratifizierten Vertrag von Lissabon gewandelt haben könnte. ${ }^{6}$

Dieses Thema soll hier anhand der Rolle von Politiknetzwerken in der europäischen Integration beispielhaft vertieft werden. In der vergleichenden Politik- und Verwaltungswissenschaft hat sich die ,policy network'-Forschung zunächst in der Analyse nationaler politischer Systeme seit den 1980er Jahren entwickelt und ist seit den 1990er Jahren zunehmend auf die Europäische Union angewandt worden. ${ }^{7}$ Bei solchen Politiknetzwerken handelt es sich nach gängigen Definitionen um überwiegend informelle, nicht oder wenig hierarchische Verbindungen und Koordinierungsmechanismen zwischen verschiedenen staatlichen (nationalen und supranationalen) und nicht-staatlichen Akteuren. Angesichts einer angeblichen ,Aushöhlung' der Nationalstaaten und ihrer dramatisch gesunkenen politischen Steuerungskompetenz haben einige Politikwissenschaftler argumentiert, dass solche Netzwerke nicht mehr nur eine wesentliche Rolle in politischen Meinungsbildungs- und Entscheidungsprozessen spielen. Vielmehr hätten sie als neue Form des Regierens beziehungsweise der ,governance ${ }^{6}$ hierarchische staatliche Strukturen abgelöst. ${ }^{8}$ Die zeitliche Dimension eines angeblichen Übergangs von hierarchischem zu Regieren in Netzwerken ist von der sozialwissenschaftlichen Forschung nicht untersucht worden, wird aber normalerweise Paul Hirst folgend in der Wirtschaftskrise der 1970er Jahre und für die heutige Europäische Union etwas später in den 1980er Jahren verortet. ${ }^{9}$ Eine solche Periodisierung haben auch Beate Kohler-Koch und Barbara Finke in ihrer jüngsten Konzeptualisierung der Beziehungen zwischen den EU-Institutionen und einer teilweise transnational verfassten europäischen ,Zivilgesellschaft‘ gewählt, die danach frühestens ab der zweiten Hälfte der 1980er Jahre als relevanter eigenständiger Akteur in Netzwerken in der EU-Politik auftaucht. ${ }^{10}$

Im Gegensatz zu der sehr spezialisierten Forschung zu ,policy networks' ist die beginnende zeithistorische Forschung zu Politiknetzwerken in einen breiteren Trend eingebettet, die jüngere europäische Geschichte stärker auch als transnationale und ,überlappende“ Geschichte zu konzeptualisieren und zu erforschen. ${ }^{11}$ Diese Forschung ist daher nicht einseitig an dem Einfluss von Politiknetzwerken auf die Ergebnisse von sektoralen Entscheidungsprozessen interessiert, sondern an verschiedenen Funktionen von - wie dies Pierre-Yves Saunier kürzlich bezeichnet hat - ,circulatory regimes“ in einem breiteren Sinne, als ,,intertextual (reading, translation, quotation) and interactional (visits, correspondence, formal and

6 Siehe für wertvolle Anregungen auch Michael Gehler: Zeitgeschichte im dynamischen Mehrebenensystem. Zwischen Regionalisierung, Nationalstaat, Europäisierung, internationaler Arena und Globalisierung, Bochum 2001.

7 Für einen guten Überblick über die verschiedenen Ansätze innerhalb dieser Forschungsrichtung siehe Eva Sørensen/Jacob Torfing (Hrsg.): Theories of Democratic Network Governance, Basingstoke 2007. Mit Bezug auf die Europäische Union siehe einführend mit weiteren Literaturhinweisen Tanja A. Börzel: Informelle Politik in Europa: Regieren in oder durch Netzwerke?, in: Michael Gehler/Wolfram Kaiser/Brigitte Leucht (Hrsg.): Netzwerke im europäischen Mehrebenensystem. Von 1945 bis zur Gegenwart, Wien/Köln/Weimar 2009, S. 27-38.

$8 \mathrm{Zu}$,governance“ in der Europäischen Union siehe unter anderem Thomas Conzelmann/Randall Smith (Hrsg.): Multi-Level Governance in the European Union: Taking Stock and Looking Ahead, Baden-Baden 2008; Beate Kohler-Koch/Berthold Rittberger: The ,governance turn' in EU studies, in: The Journal of Common Market Studies Annual Review of the European Union in 2005, Band 44, Oxford 2006, S. 27-49.

9 Paul Hirst: Democracy and Governance, in: Jon Pierre (Hrsg.): Debating Governance. Authority, Steering, and Democracy, Oxford 2000, S. 13-35.

10 Beate Kohler-Koch/Barbara Finke: The Institutional Shaping of EU-Society Relations: A Contribution to Democracy via Participation?, in: Journal of Civil Society 3/2007, S. 205-221.

11 Michael Werner/Bénédicte Zimmermann: Vergleich, Transfer, Verflechtung. Der Ansatz der ,Histoire croisée“ und die Herausforderung des Transnationalen, in: Geschichte und Gesellschaft 4/2002, S. 607-636; Thomas J. Misa/Johan Schot: Inventing Europe: Technology and the Hidden Integration of Europe, in: History and Technology $1 / 2005$, S. 1-19. 
informal organisations) communities“. ${ }^{12}$ Diese offenere Konzeptualisierung von Netzwerken führt auch dazu, dass Historiker Zweifel an dem Nutzen scharfer Abgrenzungen zwischen verschiedenen Typen von Akteuren haben. So hat Kiran Patel etwa daran erinnert, dass die katholische Kirche in historischer Perspektive kaum als staatlicher oder nicht-staatlicher Akteur eingeordnet werden kann, sondern beides war und ist. ${ }^{13}$ Für die ,policy network'-Forschung können solche für Historiker eher banalen Erkenntnisse jedoch leicht fatale Folgen haben. Nach den gängigen Definitionen ist für solche ,policy networks' nämlich konstitutiv, dass sie staatliche und nicht-staatliche Akteure umfassen: ohne nicht-staatliche Akteure keine ,policy networks' und ohne ,policy networks' kein Forschungsfeld.

Im Folgenden werden einige wesentliche Ergebnisse der laufenden zeithistorischen Forschung zu Politiknetzwerken diskutiert, die vorherrschende politikwissenschaftliche Konzepte, Annahmen und Erkenntnisse erweitern, ergänzen oder auch infrage stellen: erstens zur ,governance' der Netzwerke selbst, also deren Konstitution, ihren informellen Regeln und ihren Funktionen; zweitens zur Rolle von Netzwerken im Regieren innerhalb der heutigen Europäischen Union. Sodann sollen drittens einige Hypothesen für eine mögliche Periodisierung des relativen Einflusses von Netzwerken im Mehrebenensystem der Europäischen Union entwickelt werden.

\section{,Governance' von Netzwerken}

In der Frage, wie Politiknetzwerke ,regiert' werden, verdeutlicht die zeithistorische Forschung erstens die große Bedeutung früher Entscheidungen für deren Strukturen und, governance'-Formen, die wiederum erhebliche langfristige Folgen für die ,pfadabhängige‘ Entwicklung bestimmter Politikfelder haben können. Solche Pfadabhängigkeiten, wie dies im historischen Institutionalismus bezeichnet wird, sind gerade für die zuerst vergemeinschafteten Politiksektoren evident. So hat Brigitte Leucht gezeigt, inwieweit Kernelemente der Wettbewerbspolitik der Europäischen Wirtschaftsgemeinschaft (EWG) schon in den Anti-TrustRegelungen der EGKS angelegt waren, die wiederum stark von einem transatlantischen Netzwerk von Experten - Juristen und Ökonomen - geprägt waren. ${ }^{14}$ Leucht und Katja Seidel haben in einem gemeinsamen Beitrag herausgearbeitet, welche Kontinuitäten in diesen informellen Netzwerkstrukturen bis zur Entwicklung einer Wettbewerbspolitik durch die Europäische Kommission Anfang der 1960er Jahre bestanden haben dürften, ${ }^{15}$ was allerdings noch näher zu untersuchen wäre. Die frühen Festlegungen auf ein stark ordoliberal geprägtes Konzept von Wettbewerb und staatlicher Regulierung prägten jedenfalls die Verordnung 17/1962, ${ }^{16}$ die für 40 Jahre die gesetzliche Grundlage für die EU-Wettbewerbspolitik sein sollte, nicht zuletzt weil der deutsche Kommissar Hans von der Groeben vor allem deutsche und niederländische ordoliberal orientierte Juristen und Ökonomen als Experten konsultierte und einband. ${ }^{17}$ Selbst

12 Pierre-Yves Saunier: Learning by Doing: Notes about the Making of the Palgrave Dictionary of Transnational History, in: Journal of Modern European History 2/2008, S. 159-180.

13 Kiran K. Patel: Überlegungen zu einer transnationalen Geschichte, in: Jürgen Osterhammel (Hrsg.): Weltgeschichte, Stuttgart 2008, S. 67-89.

14 Brigitte Leucht: Transatlantic policy networks and the formation of core Europe, Dissertation University of Portsmouth 2008 .

15 Brigitte Leucht/Katja Seidel: Du Traité de Paris au règlement 17/1962: ruptures et continuités dans la politique européenne de concurrence, 1950-1962, in: Histoire, économie \& société 1/2008, S. 35-46.

16 EWG Rat: Verordnung Nr. 17: Erste Durchführungsverordnung zu den Artikeln 85 und 86 des Vertrages, in: Amtsblatt der EG, Nr. 013 vom 21. Februar 1962, S. 204-211.

17 Vgl. Katja Seidel: Administering Europe. Community Officials and the Bureaucratic Integration of Europe (1952-1967), Dissertation University of Portsmouth 2008; Sibylle Hambloch: Europäische Integration am Beispiel der Wettbewerbspolitik in der Frühphase der Europäischen Wirtschaftsgemeinschaft, Habilitation Universität Siegen 2007. 
die französischen Experten in diesem transnationalen Netzwerk unterstützten eine stärker Wettbewerb erleichternde gemeinsame Regulierung, als dies den dominanten wirtschaftspolitischen Traditionen Frankreichs und den Präferenzen seiner Regierungen entsprochen hätte. ${ }^{18}$

In ähnlicher Weise spielten frühe Entscheidungen eine zentrale Rolle für die Entstehung und weitere Entwicklung des 1983 gegründeten European Round Table of Industrialists als informelles Netzwerk von Vorständen großer Industrieunternehmen mit Hauptsitz in Europa. In Kooperation mit der Kommission und den Regierungen einiger Mitgliedstaaten, besonders Großbritanniens, beeinflusste dieser Round Table stark die Genese des Binnenmarktprogramms Anfang der 1980er Jahre. ${ }^{19}$ Die Anfänge dieses Netzwerks lagen jedoch schon in Kontakten zwischen der Kommission und europäischen Autofirmen Ende der 1960er und Anfang der 1970er Jahre, die zunächst eine Übernahme neuer, strengerer USamerikanischer Sicherheitsvorschriften verhindern wollten. ${ }^{20}$ Aus diesem Grund waren die europäischen Töchter von General Motors und Ford aus dieser Gruppe ausgeschlossen, was schließlich die Gründung des Round Table als Netzwerk ausschließlich europäischer Unternehmer ermöglichte.

Die zeithistorische Forschung unterstreicht zweitens die enorme Bedeutung der Kommission als ,gatekeeper' für die Gründung und Aufrechterhaltung von Politiknetzwerken als einer offensichtlichen Eigenart des Regierens in der Europäischen Union im Vergleich mit den Mitgliedstaaten. Wenngleich sehr schematisch hatten schon zeitgenössische neo-funktionalistische Studien auf diese zentrale Funktion hingewiesen. ${ }^{21}$ So spielte die Kommission eine große Rolle in der Transnationalisierung und Europäisierung landwirtschaftlicher Interessennetzwerke, die schon mit der Konferenz von Stresa 1958 einsetzte, die staatliche und nicht-staatliche Akteure zusammenbrachte. ${ }^{22}$ Vor allem über ihre neuen Ausschussstrukturen konnte die Kommission gesellschaftlichen Akteuren Ressourcen wie Informationen und Zugang zu Konsultationen offerieren und sie auf diese Weise zur Transnationalisierung ihrer Interessen und Aktivitäten ermuntern. Besonders seit den 1980er Jahren stellt die Kommission hierfür auch erhebliche finanzielle Mittel, zum Beispiel für die Unterhaltung von Vertretungen in Brüssel, zur Verfügung. Für die Kommission erhöhten Netzwerke unter Einschluss gesellschaftlicher Akteure nicht nur ihre technische Kompetenz und Effizienz als Institution. Vielmehr stärkte ihre Rolle als Koordinator verschiedener teilweise überlappender Netzwerke auch ihre Stellung im inter-institutionellen Wettbewerb, zunächst vor allem gegenüber den Mitgliedstaaten. Diese Rolle wurde auch nicht von de Gaulles Politik des leeren Stuhls 1965 und dem informellen sogenannten Luxemburger Kompromiss beeinträchtigt. Vor allem hat die Kommission eng in und mit europäisierten Netzwerken gearbeitet, um Steuerungsmechanismen für neue Politikfelder auf der supranationalen Ebene zu entwi-

18 Laurent Warlouzet: Quelle Europe économique pour la France? La France et le Marché commun industriel, 1956-1969, Dissertation Université Paris Sorbonne-Paris IV 2007.

19 Aus einer pluralistischen Perspektive siehe Maria Green Cowles: Setting the Agenda for a New Europe: The ERT and EC 1992, in: Journal of Common Market Studies 4/1995, S. 501-526; aus einer neo-marxistischen Perspektive vgl. Bastiaan van Apeldoorn: Transnational Capitalism and the Struggle over European Integration, London/New York 2002.

20 Vgl. Sigfrido M. Ramírez Pérez: Transnational Business Networks Propagating EC Industrial Policy: The role of the Committee of Common Market Automobile Constructors, in: Wolfram Kaiser/Brigitte Leucht/Morten Rasmussen (Hrsg.): The History of the European Union. Origins of a trans- and supranational polity 1950-72, Abingdon/New York 2009, S. 74-92.

21 Beginnend mit Ernst B. Haas: The Uniting of Europe. Political, Social, and Economic Forces, 1950-1957, Notre Dame 2004 [Original 1958].

22 Ann-Christina L. Knudsen: Farmers on Welfare. The Making of Europe's Common Agricultural Policy, Ithaca 2009. 
ckeln, für die es anfangs keine Rechtsgrundlage gab, wie Jan-Henrik Meyer derzeit in einem Projekt zur Genese der EU-Umweltpolitik in den 1970er Jahren näher untersucht. ${ }^{23}$

Die zeithistorische Forschung verdeutlicht drittens, wie wichtig es für die stark funktionalistisch und strukturalistisch ausgerichtete politikwissenschaftliche Forschung zu Netzwerken wäre, „to bring people back in“, also die Rolle einzelner Mittler in solchen Netzwerken stärker zu beleuchten. ${ }^{24}$ Sogar die stark soziologisch beeinflusste soziale Netzwerkanalyse versucht lediglich, die Beziehungen von Typen von sozialen und institutionellen Akteuren quantitativ zu erfassen und darzustellen, um auf diese Weise deren relative Zentralität im Netzwerk und ihren Einfluss auf Meinungsbildungs- und Entscheidungsprozesse zu rekonstruieren. ${ }^{25}$ Die frühe Integrationsgeschichte hatte ihre normativ-politisch geprägten Heldenepen, in denen einige ,Gründungsväter' nach 1945 scheinbar im Alleingang ein Ende der Geschichte für das nunmehr friedliche, integrierte kontinentale Westeuropa herbeiführten. Vor allem zahlreiche Arbeiten zu Jean Monnet haben dessen Rolle im frühen Integrationsprozess lange mythologisiert und die strukturellen Bedingungen für die Integration ignoriert. Jüngere Studien betonen immer noch die zentrale Rolle von Monnet und anderen handelnden Personen, nunmehr allerdings als zentrale Akteure an den Schnittstellen verschiedener überlappender westeuropäischer und transatlantischer Netzwerke für die Koordinierung der Vorbereitung und Durchführung der Regierungsverhandlungen auf der Basis des Schuman-Plans von 1950. ${ }^{26}$ In ähnlicher Weise investierten etwa die Kommissare von der Groeben und Sicco Mansholt erhebliche Ressourcen in die Entwicklung, Stabilisierung und Koordinierung transnationaler Netzwerke, um die in ihre Zuständigkeit fallenden Politikbereiche Wettbewerb, Regionalpolitik und Landwirtschaft fortzuentwickeln. ${ }^{27}$

Auffällig ist in zeithistorischer Perspektive auch, wie wichtig gerade in der Frühphase der Integration die transnationale Mittlerrolle eher unbekannter Politiker aus der zweiten Reihe oder von Privatpersonen ohne politische Ämter sein konnte. So spielte in dem informellen Genfer Kreis christdemokratischer Spitzenpolitiker Victor Koutzine, Sohn aus der Sowjetunion geflohener französischer Eltern mit exzellenten Deutschkenntnissen und enger Vertrauter Georges Bidaults aus gemeinsamen Studienzeiten an der Sorbonne, eine zentrale Rolle als Organisator und Dolmetscher, der auch persönliche Botschaften und Berichte zwischen Bonn und Paris überbrachte. ${ }^{28}$ Ein anderes Beispiel ist Alfred Mozer, der Sohn eines ungarischen Vaters und einer deutschen Mutter. Der Sozialdemokrat floh 1933 aus Deutschland und agierte nach 1945 als Internationaler Sekretär der niederländischen Partij van de Arbeid, bevor er 1958 in Mansholts Kabinett nach Brüssel wechselte. Mozer verfügte über keine nennenswerten Fachkenntnisse in Landwirtschaftsfragen, aber er spielte eine extrem wichtige Rolle in der Koordinierung vor allem von politischen Netzwerken, nicht nur zwischen Sozialdemokraten, sondern beispielsweise auch zu den deutschen Christdemokraten in den Regierungen Adenauer und Erhard, um die Entwicklung der heftig umstrittenen Gemeinsamen Agrarpolitik abzusichern. ${ }^{29}$

23 Siehe http://www.port.ac.uk/research/ceisr/members/title,78722,en.html (letzter Zugriff: 9. März 2009).

24 Siehe hierzu ausführlicher Wolfram Kaiser: Bringing People and Ideas Back in: Historical Research on the European Union, in: David Phinnemore/Alex Warleigh-Lack (Hrsg.): Reflections on European Integration. Basingstoke 2009, S. 22-39.

25 Vgl. einführend John Scott: Social Network Analysis: A Handbook, 2. Auflage, London 2000.

26 Siehe etwa Brigitte Leucht: Transatlantische Politiknetzwerke: Kulturtransfer und Schuman-Plan 1950/51, in: Comparativ 4/2006, S. 200-18.

27 Vgl. Seidel: Administering Europe, 2008.

28 Hierzu ausführlich Wolfram Kaiser: Christian Democracy and the Origins of European Union, Cambridge 2007, Kapitel 6. 
Für diese und andere transnationale Mittler waren ihre bestehenden persönlichen Kontakte und ihre interkulturelle Kompetenz wichtige Ressourcen. Ihre Fähigkeit, von einem politisch-kulturellen Kontext in einen anderen zu übersetzen, war gerade in der frühen Nachkriegszeit von herausragender Bedeutung, weil die Erfahrung totalitärer Herrschaft, des Zweiten Weltkriegs und der Besatzung und Kollaboration das ohnehin geringe soziale Kapital, vor allem Vertrauen, aufgezehrt hatte. Außerdem waren die transnationalen Kontakte wegen der geringeren Institutionalisierung und weniger entwickelten Verkehrs- und Kommunikationsinfrastruktur viel weniger intensiv und breit gefächert als in späteren Phasen fortgeschrittener Integration. Die interkulturelle Kompetenz ist jedoch bis heute eine zentrale Voraussetzung dafür geblieben, dass einzelne Akteure in EU-Institutionen und Netzwerken eine erheblich größere Rolle spielen können, als ihre übrigen Ressourcen erlaubten. Dafür ist etwa der derzeitige christdemokratische Staatsminister des kleinen Großherzogtums Luxemburg, Jean-Claude Juncker, ein exzellentes Beispiel. Juncker hat nicht nur als Mittler in den deutsch-französischen Kontakten nach dem Scheitern des Verfassungsvertrags eine wichtige Rolle gespielt, sondern etwa auch als Koordinator der informellen Eurogruppe und als engagierter Übersetzer einer im Kern föderalistischen Integrationslogik in verschiedene nationale politisch-kulturelle und mediale Kontexte. Der britische ,public policy'-Forscher Roderick Rhodes hat jüngst gefordert, solche kulturellen Dimensionen für eine ,konstruktivistische“ Analyse von Netzwerken zu berücksichtigen. ${ }^{30}$ Aus zeithistorischer Sicht besteht jedoch die Gefahr, dass die wichtige kulturelle Übersetzungstätigkeit in transnationalen Netzwerken damit rasch , unter die Räder' meta-theoretischer sozialwissenschaftlicher Richtungskämpfe zwischen konstruktivistischen und rationalistischen Forschungsansätzen geraten könnte.

Viertens verdeutlichen zeithistorische Forschungen, dass einzelne Akteure in Netzwerken normalerweise multiple Identitäten haben. Ihre Zuordnung als entweder staatliche oder nicht-staatliche beziehungsweise - mit anderen kategorialen Nuancen - öffentliche oder private Akteure mag für Sozialwissenschaftler zur Typologisierung nützlich sein. Sie erscheint jedoch nicht als geeigneter Analysefilter, vor allem nicht für Politik in der Europäischen Union als institutionell hochgradig komplexes multinationales Mehrebenensystem. So haben christdemokratische Parteiführer in ihren informellen nicht-staatlichen Parteikontakten bis zum Schuman-Plan 1950 ihre eigene Integrationspolitik als staatliche Akteure in Regierungsämtern entscheidend vorbereitet, ${ }^{31}$ wie dies Karl Magnus Johansson auch für den Maastrichter Vertrag gezeigt hat. ${ }^{32}$ Ein anderes Beispiel ist Mansholt, der nach politikwissenschaftlicher Auffassung als Kommissar Vertreter einer staatlichen, hier supranationalen Behörde war, dessen politisches Handeln jedoch zentral von seiner intensiven Einbettung in drei verschiedene transnationale Netzwerke geprägt war: ein parteipolitisch sozialdemokratisches, ein stark durch Interessengruppen und Experten geprägtes landwirtschaftspolitisches und ein ideologisch-föderalistisches im Kontext der Europäischen Bewegung.

29 Vgl. Ann-Christina L. Knudsen: Politische Unternehmer in transnationalen Politiknetzwerken. Die Ursprünge der Gemeinsamen Agrarpolitik, in: Michael Gehler/Wolfram Kaiser/Brigitte Leucht (Hrsg.): Netzwerke im europäischen Mehrebenensystem. Von 1945 bis zur Gegenwart, Wien/Köln/Weimar 2009, S. 105-120.

30 Roderick A. W. Rhodes: Putting People Back into Networks, in: Australian Journal of Political Science 3/2002, S. 399-416.

31 Kaiser: Christian Democracy, 2007, Kapitel 6. Für einschlägige Quellen siehe Michael Gehler/Wolfram Kaiser (Hrsg.): Transnationale Parteienkooperation der europäischen Christdemokraten. Dokumente 1945-1965, München 2004.

32 Karl Magnus Johansson: Another Road to Maastricht: The Christian Democrat Coalition and the Quest for European Union, in: Journal of Common Market Studies 5/2002, S. 871-893. 
Einzelne Akteure in der EU-Politik wechseln zwischen ihren Identitäten oder unterscheiden gar nicht trennscharf zwischen ihnen. Sie sprechen in unterschiedlichen informellen Netzwerken oder formellen institutionellen Kontexten verschiedene Sprachen: im übertragenen und durchaus auch im wörtlichen Sinne. Wie noch deutlich werden wird, sind diese multiplen Identitäten jedoch auch wichtig für unser Verständnis dessen, was Fritz W. Scharpf zuerst als ,Schatten der Hierarchie“ bezeichnet hat, ${ }^{33}$ den staatliche Akteure inklusive supranationaler Institutionen wie der Kommission angeblich auf das Handeln von nichthierarchischen Netzwerken werfen.

Multiple Identitäten sind dabei keinesfalls nur für Politiker wie Adenauer oder Mansholt charakteristisch. Vielmehr haben historische Soziologen wie Antoine Vauchez and Antonin Cohen diese jüngst für das sehr interessante Beispiel des Feldes (wie sie es mit Pierre Bourdieu nennen) des Europarechts untersucht. ${ }^{34}$ Für dieses juristische Netzwerk können sie eindrucksvoll zeigen, in welchem Maße dessen Mitglieder ihre professionellen Rollen wechselten oder akkumulierten. Die Mobilität von Europarechtlern im Rahmen des größeren transnationalen Netzwerkes war sehr hoch, das heißt sie waren verschiedentlich Anwälte, Richter, Professoren, Berater oder in manchen Fällen auch Generalanwälte oder Richter am Europäischen Gerichtshof. ${ }^{35}$ Diese Juristen waren jedoch Menschen mit wechselnden Anschauungen, Ideen und Gefühlslagen, die die ,governance‘ ihrer Netzwerke und ihr Handeln in ihren jeweiligen institutionellen oder funktionalen Rollen beeinflussten und somit einer genaueren empirischen Analyse bedürfen.

\section{Netzwerke im Regieren in der Europäischen Union}

Zeithistorische Forschungen zu Netzwerken in der EU-Politik erlauben zumindest einige provisorische Generalisierungen zu deren Einfluss auf Ergebnisse von Politikprozessen im Integrationskontext - dies in einem breiteren Sinne als nur bezogen auf Meinungsbildungsund Entscheidungsprozesse in einzelnen Politiksektoren.

In einer langfristigen historischen Perspektive wird erstens deutlich, in welchem Maße Netzwerke eine zentrale Rolle für die Strukturierung von geografisch und funktional differenzierten Integrationsräumen nach 1945 spielten. Es waren nämlich keinesfalls alle Netzwerke auf die Integration eines geografisch begrenzten, ökonomisch integrierten und politisch supranational organisierten Klein- oder Kerneuropa ausgerichtet. So haben die Wirtschaftshistoriker Neil Rollings und Matthias Kipping am Beispiel des 1949 gegründeten Council of European Industrial Federations gezeigt, wie sich in diesem gesamt-westeuropäischen Netzwerk in den 1950er Jahren zunehmend engere Beziehungen zwischen britischen, nordischen und schweizerischen Wirtschaftsverbänden und Industriellen entwickelten. ${ }^{36}$ In diesem Netzwerk erarbeiteten Wirtschaftsführer aus diesen Verbänden im Frühjahr 1958, als ein erfolgreicher Abschluss der laufenden Freihandelsverhandlungen immer weniger wahr-

33 Fritz W. Scharpf: Games Real Actors Could Play: Positive And Negative Coordination In Embedded Negotiations, in: Journal of Theoretical Politics 1/1994, S. 27-53. Siehe auch Adrienne Héritier/Dirk Lehmkuhl: Introduction. The Shadow of Hierarchy and New Modes of Governance, in: Journal of Public Policy 1/2008, S. 1-17.

34 Antonin Cohen: Constitutionalism Without Constitution: Transnational Elites Between Political Mobilization and Legal Expertise in the Making of a Constitution for Europe (1940s-1960s), in: Law \& Social Inquiry 1/2007, S. 109-135; Antoine Vauchez: The Force of a Weak Field: Law and Lawyers in the Government of the European Union (For a Renewed Research Agenda), in: International Political Sociology 2/2008, S. 128-144.

35 Antoine Vauchez: How to Become a Transnational Elite: Lawyers' Politics at the Genesis of the European Communities (1950-1970), in: Hanne Petersen/Anne Lise Kjær/Helle Krunke/Mikael Rask Madsen (Hrsg.): Paradoxes of European Legal Integration, Aldershot/Burlington 2008, S. 129-148.

36 Neil Rollings/Matthias Kipping: Private transnational governance in the heyday of the nation-state: the Council of European Industrial Federations (CEIF), in: Economic History Review 2/2008, S. 409-431. 
scheinlich erschien, den Plan für eine kleinere Variante ohne die EWG. Dieses Netzwerk spielte anschließend eine wichtige Rolle darin, zunächst skeptische Regierungen, vor allem die britische, von einer solchen Lösung zu überzeugen, bis diese schließlich 1959/60 die Europäische Freihandelsgemeinschaft gründeten. ${ }^{37}$

Andere transnationale Netzwerke, die schon ins 19. Jahrhundert zurückreichten, trugen wesentlich dazu bei, dass es zumindest zunächst nicht zu einer wirkungsvollen sektoralen Integration im Kontext der heutigen Europäischen Union kam. So hat etwa Johan Schot für den Transportsektor gezeigt, dass bestehende Expertennetzwerke gesamteuropäische und internationale Kooperationsformen und Regime bevorzugten. ${ }^{38}$ Trotz früher Vorschläge für eine Ausweitung der Sektorintegration auf den Transportbereich Anfang der 1950er Jahre und seiner Inklusion in den EWG-Vertrag blockierte das sektorale Expertennetzwerk lange Zeit die Herausbildung einer relevanten supranationalen EWG-Transportpolitik. Wie diese beiden Beispiele zeigen, spielten Netzwerke in der komplexen räumlichen Differenzierung transgouvernementaler und supranationaler Integration eine wichtige Rolle, was wiederum erhebliche indirekte Auswirkungen auf sektorale Politikprozesse und regulatorische Dynamiken hatte.

Zweitens legt die zeithistorische Forschung nahe, dass die Analyse von Netzwerken in bestimmten Politikfeldern die Auswirkungen konstitutionell-institutioneller Reformen und von EU-Erweiterungen auf deren Aktivitäten und ihren Einfluss erfassen sollte. Größere Politiknetzwerke mit breiteren als sektoralen Zielen und Interessen haben solche ,polity-building'-Prozesse zu steuern gesucht. Von der ersten Erweiterung der Europäischen Gemeinschaften 1973 befürchteten solche Netzwerke von Kerneuropa-Akteuren dramatische Auswirkungen, denen sie teilweise mit einer Vertiefung ihrer Kooperation in informellen Netzwerken begegnen wollten, um den politischen, acquis' insgesamt und auf einzelnen Politikfeldern zu schützen. Dass die Erweiterung der Europäischen Union um mehr oder weniger integrationsfreudige Staaten und neue gesellschaftliche Akteure erhebliche Auswirkungen auf die Interessenaggregation in Netzwerken haben dürfte, hat jüngst auch Christian Henning in einer der wenigen politikwissenschaftlichen Studien gezeigt, die solche Veränderungen systematisch über eine EU-Erweiterung hinweg untersucht haben. ${ }^{39}$ Danach haben die neuen mittel- und osteuropäischen Staaten und Interessenverbände vorerst viel stärker national ausgerichtete Netzwerke und Formen der Interessenvertretung in der Landwirtschaftspolitik. Dennoch sind die EU-Netzwerke in diesem Politikfeld deutlich stärker europäisch integriert und supranationaler geworden, weil die Ost-Erweiterungen von 2004 und 2007 in den alten Mitgliedstaaten einen starken supranationalen Integrationseffekt hatten.

Außerdem wird in zeithistorischer Perspektive drittens deutlich, dass der Einfluss von Netzwerken auf Politik und politische Entscheidungsprozesse in der Europäischen Union weder leicht kategorisiert noch gemessen werden kann. So hat Tanja Börzel vorgeschlagen, zwischen Netzwerken im Regieren und Netzwerken als neuer Form des Regierens zu unterscheiden. ${ }^{40}$ Dabei könne von einer neuen Regierungsform nur dann gesprochen werden, wenn Netzwerke politische Entscheidungen nicht nur „,vorbereiten“, sondern selbst „bestimmen“.

37 Wolfram Kaiser: Using Europe, Abusing the Europeans. Britain and European Integration, 1945-63, Basingstoke 1999, Kapitel 4.

38 Johan W. Schot: Transnational infrastructures and the origins of European integration, in: Alexander Badenoch/Andreas Fickers (Hrsg.): Europe Materializing? Transnational Infrastructures and the Project of Europe, Basingstoke im Erscheinen.

39 Christian H. C. A. Henning: Politiknetzwerke und politischer Einfluss in der EU-Agrarpolitik. Ein Vergleich der EU-15 und EU-27, in: Michael Gehler/Wolfram Kaiser/Brigitte Leucht (Hrsg.): Netzwerke im europäischen Mehrebenensystem. Von 1945 bis zur Gegenwart, Wien/Köln/Weimar 2009, S. 233-252.

40 Börzel: Informelle Politik, 2009. 
Aus Börzels skeptischer Sicht auf Netzwerke als neue Regierungsform in der Europäischen Union erscheint der ,Schatten der Hierarchie', den die Kommission durch ihr formales Monopol auf die Initiierung von Gesetzentwürfen genauso wie der Europäische Rat, der Ministerrat und teils das Europäische Parlament durch ihre Entscheidungskompetenzen werfen, lang und der politische Entscheidungsprozess nicht annähernd so nicht-hierarchisch, wie das in nationalen politischen Systemen der Fall sein mag. Allerdings ist es in zeithistorischer Perspektive empirisch kaum möglich, zwischen ,Vorbereiten' und ,Bestimmen' zu unterscheiden. So hat das Netzwerk europäischer Christdemokraten wesentliche Dimensionen der EGKS ,bestimmt ", indem es den Schuman-Plan in dieser Form ,vorbereitet' hat, nämlich mit dem Junktim zwischen supranationaler Integration und sektoraler Wirtschaftsintegration mit dem antizipierten und gewünschten Ziel des anschließenden Selbstausschlusses Großbritanniens. ${ }^{41}$

Die multiplen Identitäten einzelner Akteure in der EU-Politik erschweren darüber hinaus erheblich die empirische Beschreibung, welche Art von ,Schatten“ supranationale Institutionen in der Europäischen Union eigentlich ,werfen'. Das wird besonders anschaulich am Beispiel der ersten bahnbrechenden Entscheidung van Gend \& Loos des Europäischen Gerichtshofes von $1963,{ }^{42}$ die das Prinzip der direkten Anwendbarkeit des Gemeinschaftsrechts etablierte. Wie Morten Rasmussen rekonstruieren konnte, trafen die sieben Richter diese Entscheidung mit der knappsten möglichen Mehrheit von 4:3 Stimmen. ${ }^{43}$ Dabei kam die entscheidende Stimme ausgerechnet von dem neuen französischen Richter Robert Lecourt, der paradoxerweise im Vorjahr ausgerechnet vom französischen Präsidenten Charles de Gaulle ernannt worden war, vermutlich im Rahmen einer Abmachung mit der christdemokratischen Mouvement Républicain Populaire (MRP). Diese bildete im April 1962 für einige Wochen bis zu de Gaulles ,Volapük-Pressekonferenz' eine Koalition mit den Gaullisten. Lecourt war jedoch der frühere MRPFraktionsvorsitzende und verfügte über ausgezeichnete Kontakte im christdemokratischen Parteiennetzwerk. Er hatte stark föderalistische Präferenzen für die Verfassungsordnung Europas, weshalb er auch im Dezember 1954 in der französischen Assemblée Nationale beinahe die Pariser Verträge als unzulänglichen zwischenstaatlichen Ersatz für die supranationale Europäische Verteidigungsgemeinschaft zu Fall gebracht hätte. ${ }^{44}$ Lecourt war außerdem in der Europäischen Bewegung engagiert. Schließlich spielte er als Staatsrechtler eine wichtige Rolle in dem schon erwähnten Netzwerk von Europarechtlern. Aus dieser Perspektive erscheint die Entscheidung des Europäischen Gerichtshofes von 1963 als ein dramatischer Sieg mit erheblichen langfristigen Auswirkungen auf das politische System der heutigen Europäischen Union für transnationale parteipolitische, föderalistische und juristische Netzwerke in einem hochgradig politisierten Konflikt über die Verfassungsordnung und Zukunft der EWG - keinesfalls als ein ,Schatten', den eine staatliche supranationale Institution auf Netzwerke ,warf'.

\section{Von Netzwerken im Regieren zu ,network governance'?}

In der Frage einer Periodisierung von Formen des Regierens in der heutigen Europäischen Union könnten es sich Historiker leicht machen. Wie immer ist ihnen längst aus vergangenen Jahrhunderten bekannt, was Politikwissenschaftler gerade als neuen Trend - in diesem Fall zur ,network governance ${ }^{45}$ - ausgerufen haben. Natürlich wurde in der Antike, im Mittelalter und in der frühen Neuzeit und außerdem in anderen geografischen Räumen länger als in Europa

41 Vgl. Kaiser: Christian Democracy, 2007, Kapitel 6.

42 Europäischer Gerichtshof: Rs. 26/62 (van Gend \& Loos/Niederländische Finanzverwaltung), Slg. 1963, 3.

43 Morten Rasmussen: From Costa v. ENEL to the Treaties of Rome - A Brief History of a Legal Revolution, in: Luís Miguel Poiares Maduro/Loïc Azoulai (Hrsg.): The past and Future of EU law. The classics of EU Law Revisited on the $50^{\text {th }}$ Anniversary of the Rome Treaty, Oxford im Erscheinen.

44 Vgl. Kaiser: Christian Democracy, 2007, Kapitel 7. 
ganz überwiegend in Netzwerken und Märkten ,regiert‘. So wie der Nationalstaat des 19. Jahrhunderts könnte sich im Übrigen auch der hierarchische hochgradig regulierte Wohlfahrtsstaat in Westeuropa nach 1945, von dem Politikwissenschaftler das angeblich neue Regieren in Netzwerken abgrenzen, in der ,longue durée' noch als kurze historische Episode erweisen.

Doch wie hierarchisch war der nationale Wohlfahrtsstaat überhaupt als Akteur in der Integrationspolitik nach 1945? Selbst einige Politikwissenschaftler haben jüngst aggressiv die Annahme hinterfragt, dass es irgendwann einen Wechsel von ,Hierarchie‘ zu ,Regieren“ in Netzwerken gegeben habe. So hat Michael Keating in Bezug auf britische ,policy network'Literatur konstatiert, die ,network governance'-These basiere letztlich auf einem ,stilisierten Narrativ“ einer ,fiktionalen Welt vor ,governance“, in der es noch unitarisch verfasste, zentralisierte Staaten gab, die irgendwie autonom von sozialen Interessen existierten" ${ }^{46}$ Für einen föderalistischen Staat wie die Bundesrepublik Deutschland wäre ein solches Narrativ ohnehin abwegig. Als Akteure im Integrationskontext erweisen sich in zeithistorischer Perspektive jedoch selbst zentralisierte Mitgliedstaaten als stark fragmentiert. Politische Parteien, Koalitionsregierungen, nationale Bürokratien und Verbände waren in wesentlichen strategischen Kernfragen der Integration tief gespalten und suchten Verbündete für ihre Präferenzen nicht nur unter anderen nationalen Akteuren, sondern vor allem auch in transnationalen Netzwerken und Allianzen und in den supranationalen Institutionen.

Selbst den französischen Regierungen in der V. Republik fiel es in dem zunehmend pluralistischen, multinationalen Mehrebenensystem schwer, klare Präferenzen zu formulieren und Einfluss zu projizieren, wie beispielsweise die enge Kooperation liberaler Fachbeamter in den Pariser Wirtschaftsministerien mit den Ministerien anderer Mitgliedstaaten und der Kommission oder die dramatischen Folgen der Ernennung Lecourts zum Richter am Europäischen Gerichtshof illustrieren. Von Anfang an schufen die supranationalen Behörden für Experten aus Wissenschaft, gesellschaftlichen Organisationen und Verbänden Zugang zu Meinungsbildungs- und Entscheidungsprozessen. Die zahlreichen historischen Beispiele für netzwerkartige informelle Koordinierung, auch unter Einschluss nicht-staatlicher Akteure, aus der Zeit vor der ersten EG-Erweiterung 1973 verdeutlichen, dass es in der Europäischen Union niemals einen abrupten Wandel von hierarchischem zum Regieren in Netzwerken gegeben hat. Genauso wenig sollte man sich einen solchen Wandel als linear zunehmende Verdichtung von Netzwerken und - damit einhergehend - deren kontinuierlich immer größeren Einfluss auf Entscheidungsprozesse hin zu einer neuen Form von Regieren vorstellen. Zum Beispiel kann es in einer globalen Wirtschaftskrise, wie sie 2008 einsetzte, zu erheblichen Verwerfungen innerhalb von transnationalen Netzwerken sowie zu einer zumindest vorübergehenden Wiederbelebung stark staatlich-hierarchischer Entscheidungsstrukturen kommen.

Wie ließe sich dann Wandel in Netzwerken in der Europäischen Union alternativ konzeptualisieren? Insofern erscheint die von Mark Bevir und Roderick Rhodes allerdings nicht speziell auf die Europäische Union bezogene Hypothese bedenkenswert, dass politische „Dilemmata“ als Katalysatoren zumindest einen solchen Wandel in Netzwerken auslösen können, wenngleich damit nicht notwendigerweise auch in ihrem Einfluss auf das Regieren innerhalb der Europäischen Union. Solche Dilemmata stellen die Konstitution und Aktivitä-

45 Andrew Jordan/Adriaan Schout: The coordination of European Governance: exploring the capacities for networked governance, Oxford 2006; Adriaan Schout/Andrew Jordan: Coordinated European Governance. Self-Organizing or Centrally Steered?, in: Public Administration 1/2005, S. 201-220; Beate Kohler-Koch/Rainer Eising (Hrsg.): The Transformation of Governance in Europe, London 1999; Beate Kohler-Koch: Catching up with Change: The Transformation of Governance in the European Union, in: Journal of European Public Policy 3/1996, S. 359-380.

46 Michael Keating: Thirty Years of Territorial Politics, in: West European Politics 1-2/2008, S. 60-81, hier S. 76. Eigene Übersetzung. 
ten von Politiknetzwerken, bisherige Traditionen des Regierens und etablierte Lösungen für einzelne Politikfelder infrage. ${ }^{47}$ Auf einer allgemeinen Ebene, nicht bezogen auf spezifische Politikfelder, erscheinen drei Arten von Dilemmata als besonders signifikant, die die Transformation existierender und die Formierung neuer Netzwerke angestoßen und Phasen besonders intensiver, vernetzter' Politik eingeleitet haben. Das betrifft erstens konstitutionellinstitutionellen Wandel einschließlich von EU-Erweiterungen. So schuf die Gründung der EGKS und der EWG eine gänzlich neue institutionelle Ordnung, in der die Rolle der supranationalen Institutionen und gesellschaftlichen Akteure erst einmal bestimmt werden musste. Dies war für sich genommen ein großer Anreiz, bestehende Politiknetzwerke zu aktivieren oder neue zu schaffen, um die vertraglich teils nur sehr vage beschriebenen gesetzgeberischen Aufgaben erfüllen zu können. Die größte Verfassungsreform der Europäischen Union erfolgte sodann im Maastrichter Vertrag von 1992, der vor allem zu einer dramatischen Aufwertung des Europäischen Parlaments als Akteur in Netzwerken sowie als Lokus zivilgesellschaftlicher Verständigungsprozesse führte.

Neben diesen Ergebnissen zwischenstaatlicher Verhandlungen über neue Verträge beziehungsweise Vertragsrevisionen schufen auch EU-Erweiterungen Dilemmata. Das gilt besonders für solche, die entweder die im Wesentlichen föderalistische Stoßrichtung Kerneuropas oder dessen innere politische und ökonomische Balance durch eine ganz neue Stimmengewichtung und erhebliche Budgettransfers wesentlich infrage zu stellen schienen. So hatte vermutlich der Beitritt Großbritanniens eine Stärkung bestehender allgemeiner und sektoraler Politiknetzwerke zur Folge, um - um im Bild zu bleiben - die befürchtete Kernschmelze in der Integration abzuwenden.

Zweitens ergeben sich große Dilemmata aus ökonomischen und gesellschaftlichen Krisenerscheinungen, die zu gesteigerten Erwartungen an die Problemlösungskompetenz der Europäischen Union führen und eine größere Koordinierung mitgliedstaatlicher Politik erfordern beziehungsweise eine Europäisierung von Politikfeldern angestoßen haben. Dies gilt vor allem für die Zeit nach der Ölkrise von 1973, die in den Europäischen Gemeinschaften und darüber hinaus von geringem Wachstum, wachsender Arbeitslosigkeit und rasch steigenden Budget- und Staatsdefiziten geprägt war. Bis dahin hatten die meisten Wirtschaftsverbände und Unternehmen die Integration grundsätzlich befürwortet und kaum Lobbying betrieben, zumal die Kommission Wert darauf legte, nicht als verlängerter Arm der Industrie zu erscheinen. Die Wirtschafts- und Energiekrise, sich teilweise dramatisch zuspitzende Verteilungskämpfe und wachsende Sorgen über die internationale Wettbewerbsfähigkeit der europäischen Wirtschaft im Vergleich mit Japan und den Vereinigten Staaten führten nun jedoch dazu, dass die Unternehmen eigene Netzwerke auf- und ausbauten. Sie schalteten sich auch zunehmend öffentlich in Diskussionen über Reformen in den Europäischen Gemeinschaften ein, besonders im Vorfeld der Gründung des European Round Table of Industrialists und seiner Initiative für die Vollendung des Binnenmarkts.

Das dritte für die Konstituierung und Transformation von Netzwerken besonders relevante Dilemma besteht in Legitimitätskrisen der Integration. Diese haben in der Vergangenheit vor allem dazu geführt, dass supranationale Institutionen sich verstärkt um die Aktivierung und Einbeziehung gesellschaftlicher Akteure in bestehende formelle Konsultationsmechanismen und informelle Netzwerke bemüht haben - dies vor allem, weil sie sich davon größere ,Input'-Legitimität erhofft haben. Zwar waren die 1970er Jahre noch von einem recht linear zunehmenden permissiven Konsens in der Integration geprägt, das heißt ei-

47 Mark Bevir/Roderick A. W. Rhodes: Decentred Theory, Change and Network Governance, in: Eva Sørensen/ Jacob Torfing (Hrsg.): Theories of Democratic Network Governance, Basingstoke 2007, S. 77-91. 
ner wachsenden generellen Unterstützung durch die Bürger selbst der neuen Mitgliedstaaten, verbunden mit weitgehendem Desinteresse an konkreten europapolitischen Themen. Wie Ian Down und Carole Wilson jedoch mit Recht betont haben, waren die 1970er Jahre viel mehr als die 1990er Jahre eine Zeit extremer „Polarisierung“ in Integrationsfragen mit einer minoritären, aber öffentlich aggressiv artikulierten Opposition jedenfalls gegen diese Art von Integration. ${ }^{48} \mathrm{Zu}$ dieser Zeit betrachteten die Studentenbewegung und politisch links stehende Protestbewegungen die Europäischen Gemeinschaften als die supranationale Verkörperung kapitalistischer ,Ausbeutung'. Die neuen sozialen Bewegungen stellten etablierte Formen institutioneller Politik nicht nur auf der nationalen, sondern auch auf der europäischen und internationalen Ebene infrage. Wie erneut ab der zweiten Hälfte der 1990er Jahre bemühte sich die Kommission, solche Unzufriedenheit einzudämmen, indem sie den neuen gesellschaftlichen Akteuren behilflich war, transnationale Kontakte zu entwickeln und zu formalisieren, und sie in die Entwicklung neuer Politikfelder wie der Umweltpolitik einbezog.

Insofern erscheint die Annahme vertretbar, die aber möglichst durch interdisziplinäre Forschung zu erhärten wäre, dass es in der europäischen Integration seit 1945 eine starke Verdichtung von Netzwerken im EU-Regieren in solchen Phasen gegeben hat, in denen konstitutionell-institutionelle Reformen einschließlich der EU-Erweiterungen sowie ökonomisch-gesellschaftliche Krisenerscheinungen bis dahin gültige formelle und informelle institutionelle Arrangements radikal infrage gestellt haben. Das war nach der EGKSGründung vor allem in der Anfangszeit der EWG zwischen 1958 und etwa 1963, erneut von der Ölkrise 1973 bis Anfang der 1980er Jahre und wieder ab 1992 der Fall. Außerdem haben Legitimitätskrisen vor allem in der Zeit nach 1973 und 1992 zu einer stärkeren Pluralisierung von Akteuren in Netzwerken, vor allem zur Aktivierung von Wirtschaftsakteuren in den 1970er Jahren und von zivilgesellschaftlichen Akteuren in den 1990er Jahren geführt. Dagegen erscheint das rasche quantitative Wachstum von Interessenrepräsentation in Brüssel im Kontext des Binnenmarktprogramms Ende der 1980er Jahre keine neue Phase der Bildung und Intensivierung von Netzwerken oder gar von Regieren in und durch Netzwerke zu markieren, sondern eine Transformation von Praktiken des Lobbying, das stärker auf die supranationale Ebene verlagert wurde. ${ }^{49}$ Aus einer solchen Perspektive erscheinen die 1970er Jahre, die in der Integrationsgeschichte früher als Phase politisch-institutioneller Stagnation gesehen wurden, durchaus als eine wichtige Sattelzeit ${ }^{50}$ für Netzwerke, doch kommen auf EU-Ebene drei verschiedene Dilemmata oder Faktoren zusammen, die zu ihrer Neugründung, Transformation, Integration und Pluralisierung beitragen. Die These einer ,Aushöhlung' der nationalen Mitgliedstaaten, wie sie ,policy network'-Forscher für den nationalstaatlichen Kontext entwickelt haben, greift jedenfalls für die heutige Europäische Union in zeithistorischer Perspektive gleich in mehrfacher Hinsicht zu kurz.

\section{Schluss}

Die zeithistorische Forschung zu Netzwerken und informeller Koordinierung in der Politik der heutigen Europäischen Union steckt noch in den Anfängen. Sie wird nicht zuletzt da-

48 Ian Down/Carole J. Wilson: From ,Permissive Consensus' to ,Constraining Dissensus': A Polarizing Union?, in: Acta Politica 1/2008, S. 26-49.

49 Vgl. hierzu einführend Justin Greenwood: Interest Representation in the European Union, 2. Auflage, Basingstoke 2007.

50 Reinhart Koselleck: Einleitung, in: Otto Brunner/Werner Conze/Reinhart Koselleck (Hrsg.): Geschichtliche Grundbegriffe. Historisches Lexikon zur politisch-sozialen Sprache in Deutschland, Band 1, Stuttgart 1972, S. XIII-XXVII, hier S. XV. 
durch erschwert, dass die hierfür notwendige multinationale, multi-institutionelle und multiarchivische Forschung sehr zeitaufwendig ist. Außerdem ist informelle mündliche Koordinierung nur bedingt mithilfe vor allem im Falle gesellschaftlicher Akteure bestenfalls zufällig überlieferter schriftlicher Quellen rekonstruierbar. Der analytische Blick auf vernetzte Politik wird den Zeithistorikern jedoch vor allem dabei behilflich sein, die Fragmentierung der Mitgliedstaaten und supranationalen Institutionen als Akteure und den informellen Charakter der Generierung und Aushandlung von politischen Ideen und Konzepten in transnationalen politischen Kontexten besser zu verstehen.

Die hier aufgeführten Beispiele illustrieren jedoch darüber hinaus, dass diese zeithistorische Netzwerk-Forschung nicht nur die stark national staatszentrierte traditionelle Integrationsgeschichte perspektivisch um die transnationale Dimension von Politik in der Europäischen Union erweitern und so auch zu ihrer besseren Integration in die allgemeine Geschichtsschreibung Europas beitragen kann. Vielmehr hat diese Forschung auch Potenzial, die Ergebnisse einer sehr eng geführten politikwissenschaftlichen ,policy network'Forschung zu erweitern und kritisch zu hinterfragen. Vor allem kann diese zeithistorische Forschung helfen, die ,policy network'-Forschung wieder stärker an Verfassungsprozesse in einer transnationalen politischen Gemeinschaft anzubinden. Sie kann neue empirisch untermauerte Einsichten in die Bedingungen erarbeiten, unter denen neue Netzwerke entstehen und alte sich transformieren, während die meiste politikwissenschaftliche Forschung erst einsetzt, wenn solche Netzwerke schon sichtbaren Einfluss auf Entscheidungsprozesse haben. Schließlich kann die zeithistorische Forschung auch in diachroner Perspektive simplifizierende Annahmen über den Wandel von Staatlichkeit und einen Wechsel von hierarchischem Regieren zu Regieren in Netzwerken modifizieren. Eine engere transdisziplinäre Kooperation zwischen der Zeitgeschichte und der Politikwissenschaft hätte vor allem großes Potenzial, Wandel im Regieren in der Europäischen Union besser zu verstehen, vor allem hinsichtlich der Entstehung, Abschließung und Öffnung solcher Netzwerke in verschiedenen Politikfeldern, der Rolle supranationaler Institutionen wie der Kommission als Initiatoren und Mittler in solchen Netzwerken und des Einflusses von Netzwerken auf die EU-Politik. Auf diese Weise kann die Zeitgeschichte in Kooperation mit der Politikwissenschaft neue Wege zu einer transdisziplinären Rekonstruktion von zeitlichem Wandel nicht nur von transnationalen Netzwerken im Regieren in der Europäischen Union, sondern auch in der europäischen Integration insgesamt aufzeigen.

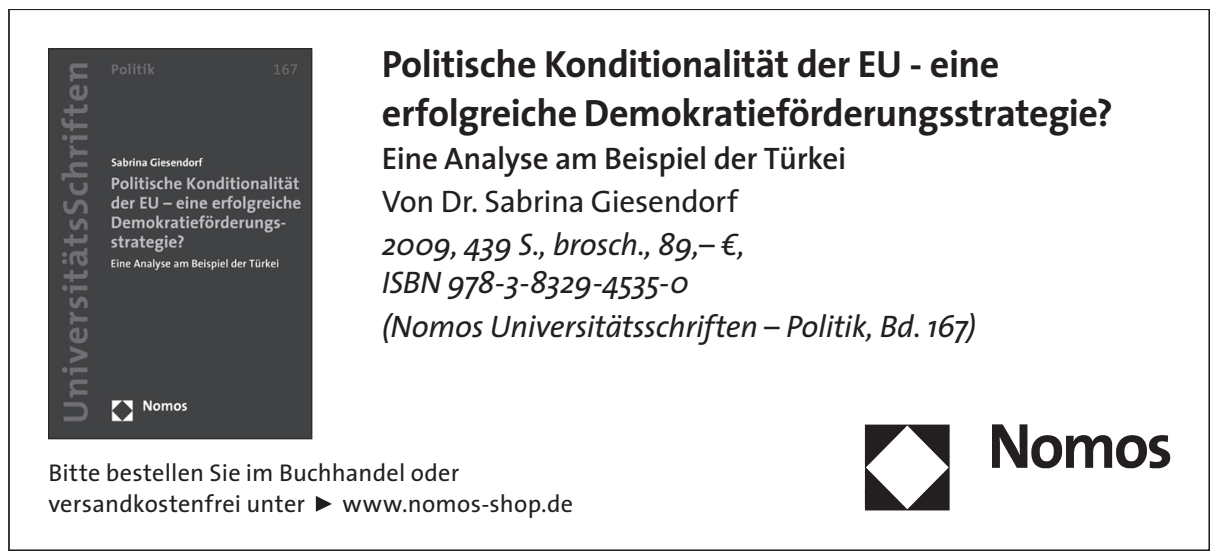

